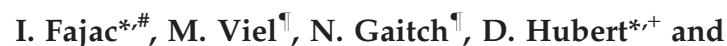

T. Bienvenu ${ }^{\top, \S, f}$

*Université Paris Descartes, "Service d'Explorations Fonctionnelles, Assistance Publique - Hôpitaux de Paris, Hôpital Cochin, "Laboratoire de Biochimie et Génétique Moléculaires, Hôpital Cochin, 'Service de Pneumologie, Assistance Publique Hôpitaux de Paris, Hôpital Cochin, ${ }^{\S}$ Université Paris Descartes, Institut Cochin, CNRS (UMR 8104), and ${ }^{f}$ Inserm U567, Paris, France.

Correspondence: T. Bienvenu, Laboratoire de Biochimie et Génétique Moléculaires, Hôpital Cochin, 123 boulevard de Port Royal, 75014, Paris, France. E-mail: thierry.bienvenu@ inserm.fr

Statement of Interest: None declared.

\section{REFERENCES}

1 Fajac I, Viel M, Sublemontier S, et al. Could a defective epithelial sodium channel lead to bronchiectasis. Respir Res 2008; 9: 46-53.
2 Azad AK, Rauh R, Vermeulen F, et al. Mutations in the amiloridesensitive epithelial sodium channel in patients with cystic fibrosislike disease. Hum Mutat 2009; 30: 1093-1103.

3 Samaha FF, Rubenstein RC, Yan W, et al. Functional polymorphism in the carboxyl terminus of the $\alpha$-subunit of the human epithelial sodium channel. J Biol Chem 2004; 279: 23900-23907.

4 Tong Q, Menon AG, Stockand JD. Functional polymorphisms in the $\alpha$-subunit of the human epithelial $\mathrm{Na}^{+}$channel increase activity. Am J Physiol Renal Physiol 2006; 290: F821-F827.

5 Sugiyama T, Kato N, Yamori Y, et al. Evaluation of selected polymorphisms of the mendelian hypertensive disease genes in the Japanese population. Hypertens Res 2001; 24: 515-521.

6 Des Georges M, Guittard C, Altieri JP, et al. High heterogeneity of CFTR mutations and unexpected low incidence of cystic fibrosis in the Mediterranean France. J Cyst Fibros 2004; 3: 265-272.

7 Chillon M, Casals T, Mercier B, et al. Mutations in the cystic fibrosis gene in patients with congenital absence of the vas deferens. $N$ Engl J Med 1995; 332: 1475-1480.

8 Mutesa L, Azad AK, Verhaeghe C, et al. Genetic analysis of Rwandan patients with cystic fibrosis-like symptoms: identification of novel cystic fibrosis transmembrane conductance regulator and epithelial sodium channel gene variants. Chest 2009; 135: 1233-1242.

DOI: $10.1183 / 09031936.00057309$

\title{
Diagnosis of a posterior mediastinal goitre via endobronchial ultrasound-guided transbronchial needle aspiration
}

\section{To the Editors:}

Endobronchial ultrasound-guided transbronchial needle aspiration (EBUS-TBNA) is a rapidly developing diagnostic tool. Its utility as a minimally invasive and safe procedure in mediastinal node staging in lung cancer is defined and gaining widespread acceptance. The role of this procedure in the diagnosis of benign diseases is less clear. We report the first case of a posterior mediastinal intrathoracic goitre diagnosed with the aid of EBUS-TBNA.

A 46-yr-old female was referred to the outpatient chest clinic for further investigation of a superior mediastinal mass. She complained of a 4-month history of progressive shortness of breath on exertion. She denied any dysphagia. Examination was unremarkable. A chest radiograph showed a right superior mediastinal mass. Her thyroid function tests were within normal limits. Contrast-enhanced thoracic computed tomography revealed a large posterior mediastinal mass, containing areas of punctate calcification (fig. 1). There was no associated mediastinal adenopathy and the lung parenchyma was normal. Histological confirmation was considered necessary to the planning of surgical intervention. Oesophageal endoscopic ultrasound-guided needle aspiration was abandoned, as the scope was unable to pass down the oesophagus due to extrinsic compression. We performed endobronchial ultrasound guided transtracheal needle aspiration of the posterior mediastinal mass under conscious sedation, using a linear array ultrasonic bronchoscope and a 22-gauge needle. A large heterogeneous mass was identified adjacent to the posterior wall of the upper trachea. Three separate passes into the mass were performed. The procedure was well tolerated with no complications. Cytology revealed low cuboidal and columnar epithelium with follicle formation containing small amounts of colloid. Thyroglobulin (fig. 2) and thyroid transcription factor 1 stains were positive, confirming thyroid origin. A diagnosis of posterior mediastinal intrathoracic goitre was made. The patient was referred for total thyroidectomy, which was successfully performed using a transcervical approach. The histology was in keeping with a benign multinodular goitre with areas of cystic change, old and recent haemorrhage, fibrosis and patchy calcification. No evidence of malignancy was found.

The differential diagnosis of a posterior mediastinal mass is wide and includes neurogenic tumours, Castleman disease, bronchogenic cysts, Bochdalek hernia, mesenchymal tumours and intrathoracic goitre. The radiological features of a goitre include: heterogeneous attenuation, focal punctate or curvilinear calcification within the mass, rapid and prolonged enhancement of the mass after contrast injection, and continuity of the mass with one of the lobes of the thyroid gland [1].

The majority of intrathoracic goitres are acquired and arise from the cervical thyroid gland. In 1934, LAHEY and SWINTON [2] proposed anatomic factors that facilitate cervical goitres to extend downward into the mediastinum through the thoracic inlet as 

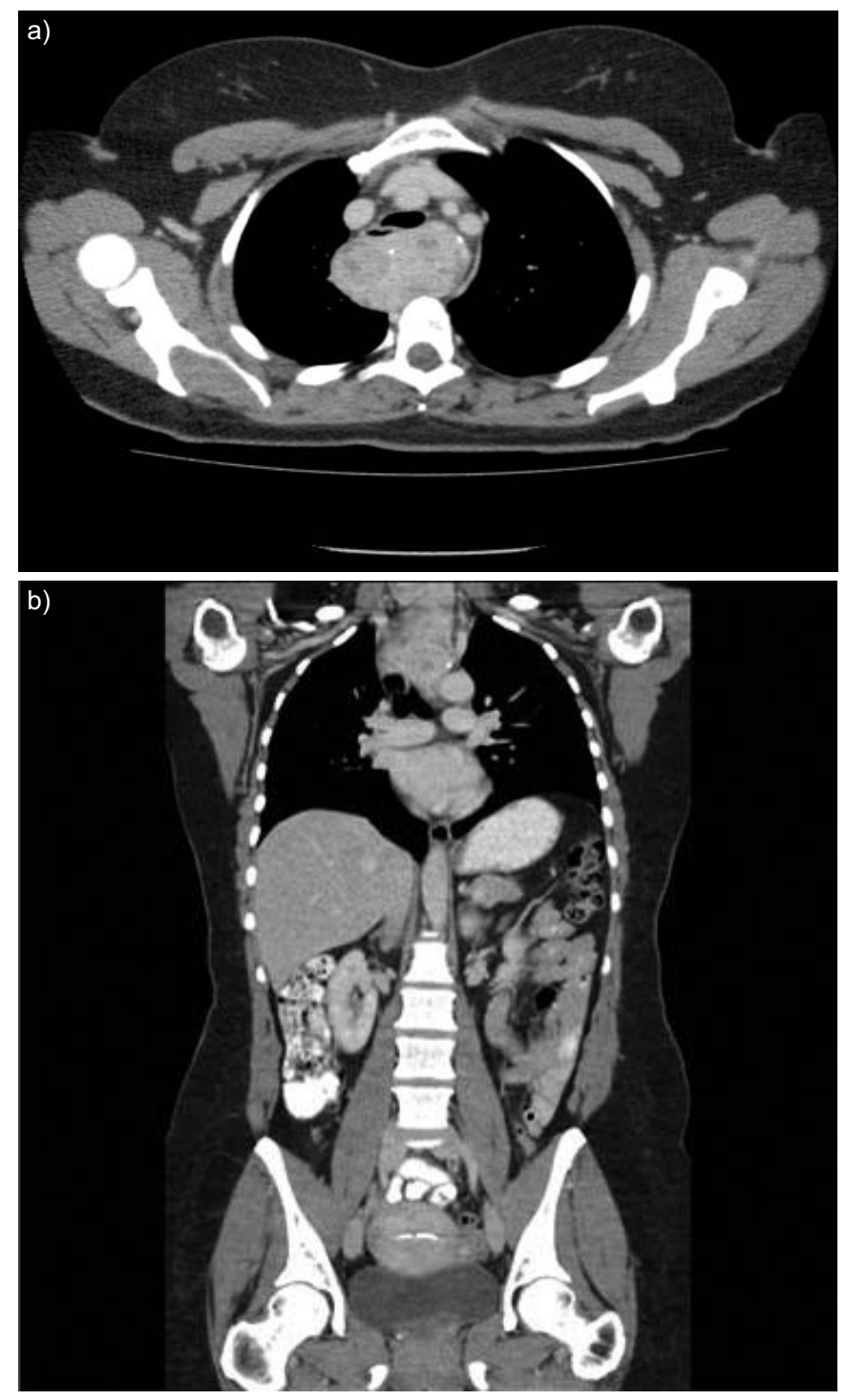

FIGURE 1. a) Contrast-enhanced computed tomography (CT) showing a large retrotracheal and retro-oesophageal mass with heterogeneous enhancement and punctate calcification. b) Coronal view of the thorax CT showing mediastinal mass in continuity with left lobe of thyroid gland.

they grow. These include gravity, the absence of fasciae inferiorly, the weight of the goitre and the intrathoracic pressure changes with respiration and swallowing. Most intrathoracic goitres are pre-tracheal and retrosternal. However, posterior mediastinal location of the goitres has been reported, with up to $15 \%$ of cases of intrathoracic goitres [3] being predominantly retrotracheal and, less frequently, as in our case, retro-oesophageal. More rarely, extension of the goitres to the contralateral side of origin in the cervical goitre has been reported [4]. Less than $1 \%$ of surgically removed goitres are aberrant thyroid tissues, which can be found anywhere in the mediastinum, with no apparent connection with the cervical goitre. These are congenital in nature and derive their blood supply directly from the intrathoracic vessels [4].

Given the progressive nature of the goitres, even in the absence of symptoms, early surgical resection is recommended, unless

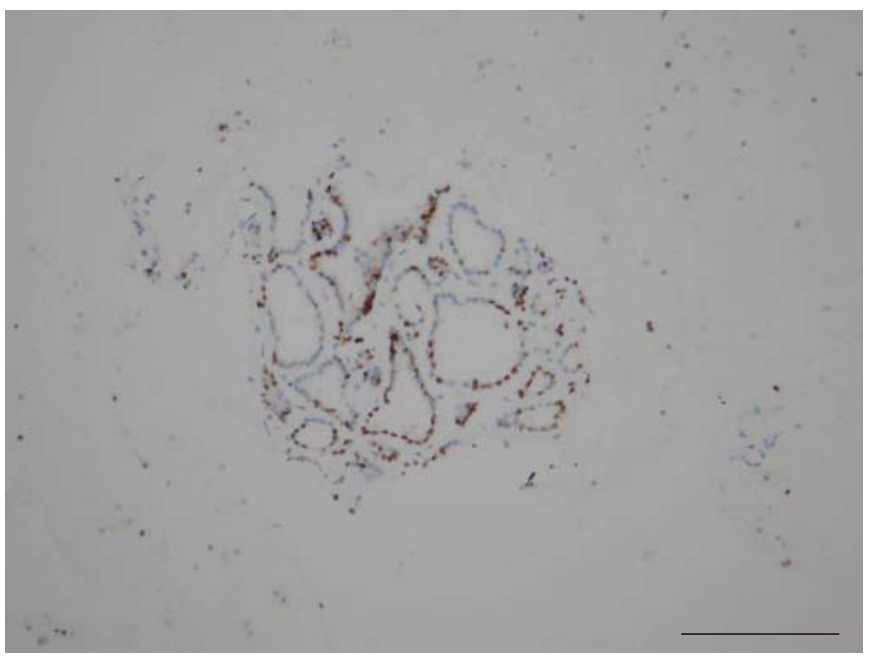

FIGURE 2. Endobronchial ultrasound-guided transbronchial needle aspiration specimen of posterior mediastinal mass showing uptake of follicles with thyroglobulin stain. Scale bar=1.5 $\mathrm{mm}$.

the patient is deemed unfit [5]. The most pressing indications include the compression of adjacent organs, including the trachea, oesophagus and superior vena cava. Cases of sudden enlargement of the intrathoracic goitres with respiratory compromise have been reported and are usually due to haemorrhage into the cystic lesions or infection within the mass [6, 7]. Surgery also enables a tissue diagnosis. It is noteworthy that small foci of papillary microcarcinoma can be found within the intrathoracic goitres [8].

In cases of diagnostic doubt, particularly when surgery is being considered, histological confirmation may be needed. So far, the role of EBUS-TBNA has been predominantly centred towards the sampling of mediastinal and hilar nodes to stage lung cancer [9] and to sample peri-bronchial tumours. Increasingly, however, the technique has been used to diagnose benign disease, notably sarcoidosis [10]. To our knowledge, this is the first report of its use in the diagnosis of a posteriorly situated mediastinal goiter.

Our report highlights that endobronchial ultrasound should be considered in the diagnostic algorithm when evaluating an unexplained posterior mediastinal mass.

\section{Jeebun*, S. Natu ${ }^{\#}$ and R. Harrison*}

*Respiratory Dept and "Dept of Pathology, University Hospital of North Tees, Stockton-on-Tees, UK.

Correspondence: V. Jeebun, Respiratory Dept, University Hospital of North Tees, Stockton-on-Tees, TS19 8PE, UK. Email: vandana.jeebun@nth.nhs.uk

Statement of Interest: None declared.

\section{REFERENCES}

1 Bashist B, Ellis K, Gold RP. Computed tomography of intrathoracic goiters. AJR Am J Roentgenol 1983; 140: 455-460. 
2 Lahey FH, Swinton NW. Intrathoracic goiter. Surg Gynecol Obstet 1934; 59: 627-637.

3 Chin SC, Rice H, Som PM. Spread of goiters outside the thyroid bed: a review of 190 cases and an analysis of the incidence of the various extensions. Arch Otololaryngol Head Neck Surg 2003; 129: 1198-1202.

4 Hilton HD, Griffin WT. Posterior mediastinal goiter. Am J Surg 1968; 116: 891-895.

5 Hashmi SM, Premachandra DJ, Bennett AMD, et al. Management of retrosternal goitres: results of early surgical intervention to prevent airway morbidity, and a review of the literature. J Laryngol Otol 2006; 120: 644-649.

6 Ket S, Ozbudak O, Ozdemir T, et al. Acute respiratory failure and tracheal obstruction in patients with posterior giant mediastinal (intrathoracic) goiter. Interact Cardiovasc Thorac Surg 2004; 3: 174-175.
7 Karadeniz A, Hacihanefioglu U. Abscess formation in an intrathoracic goitre. Thorax 1982; 37: 556-557.

8 Shah BC, Ravichand CS, Juluri S, et al. Ectopic thyroid cancer. Ann Thorac Cardiovasc Surg 2007; 13: 122-124.

9 Herth FJ, Rabe KF, Gasparini S, et al. Transbronchial and transoesophageal (ultrasound-guided) needle aspirations for the analysis of mediastinal lesions. Eur Respir J 2006; 28: 1264-1275.

10 Wong M, Yasufuku K, Nakajima T. Endobronchial ultrasound new insight for the diagnosis of sarcoidosis. Eur Respir J 2007; 29: 1182-1186.

DOI: $10.1183 / 09031936.00046309$ 